\title{
Investigation of the Nature of Capping Layer Materials for FIB-SEM Preparation: Implications for the Study of Carbonaceous Material in Extraterrestrial Samples
}

\author{
Pierre Haenecour ${ }^{1}$, Thomas J. Zega ${ }^{1,2}$, Jane Y. Howe ${ }^{3}$, Paul Wallace ${ }^{1}$, Christine Floss ${ }^{4}$, and Toru Yada ${ }^{5}$ \\ 1. Lunar and Planetary Laboratory, The University of Arizona, Tucson, USA. \\ 2. Dept. of Materials Science and Engineering, The University of Arizona, Tucson, USA. \\ 3. Hitachi High-Technologies America Inc., Clarksburg, USA. \\ 4. Laboratory for Space Sciences and Physics Department, Washington University, St. Louis, USA. \\ 5. JAXA Institute of Space and Astronautical Science, Sagamihara, Japan.
}

The application of transmission electron microscopy (TEM) and electron energy-loss spectroscopy (EELS) to planetary materials has allowed study of the nature, microstructure and the functional chemistry of refractory polymeric materials found in meteorites, interplanetary dust particles, and samples returned from NASA missions (e.g., Stardust) [1, 2]. However, these techniques require the preparation of electron-transparent cross-sections ( $<100 \mathrm{~nm}$ in thickness) of the region of interest [3]. The development of focused-ion beam (FIB) has revolutionized our ability to carry out TEM analysis of extraterrestrial material, allowing us to study such materials in situ in their native context [3, 4]. To protect the region of interest from the ion beam during the cross-section extraction, a protective capping layer must be deposited on the sample's surface (typically $\mathrm{C}$ or Pt on dual beam FIBs). However, it is unclear if the use of $\mathrm{C}$ as a capping layer could present problems (e.g., $\mathrm{C}$ re-deposition) for the investigation of $\mathrm{C}$-bearing low-Z materials. In particular, this is an important question for our current work on isotopically anomalous organic matter. Using a coordinated multi-technique approach (e.g., NanoSIMS, TEM, EELS), we are investigating the distribution and chemical functional groups of the carrier(s) of nitrogen and hydrogen isotopically anomalies associated with organic matter in carbonaceous chondrites, as well as their possible relationship with other surrounding matrix materials. These anomalies, also referred to as 'hotspots', are identified in 2-D NanoSIMS raster ion maps of the samples. Here, we report preliminary data on the distribution of carbon and carbon-functional chemistry in a cross-section of a fine-grained Antarctic micrometeorite that was prepared using a $\mathrm{C}$ capping layer that allow us to investigate this question.

The micrometeorite (MM), T00IBa030, was collected by melting and filtering blue ice at Tottuki Point in East Antarctica [5]. We carried out ion imaging of $\mathrm{C}$ and $\mathrm{N}$ isotopes using the Cameca NanoSIMS 50 to identify $\mathrm{N}$ anomalous hotspots. We then extracted a cross-section of a random area (without any hotspot) in the MM using the FEI Helios FIB scanning electron microscope. A C-capping layer $(8 \times 1.5$ $\times 3 \mu \mathrm{m}$ ) was deposited onto the selected region using both electron- (at $2 \mathrm{kV} \& 3.2 \mathrm{nA}$ ) and ion-beam deposition (at $30 \mathrm{kV} \& 243 \mathrm{pA}$ ). The section was then cut and thinned using the FEI easylift micromanipulator, over a range of voltages and currents. An initial coarse cut was performed at $30 \mathrm{keV}$ and $9.3 \mathrm{nA}$ to a sample thickness of $1 \mu \mathrm{m}$. We then lowered the current to $2.5 \mathrm{nA}$ for a fine cut and then to $0.79 \mathrm{nA}$ for the undercut. After mounting the section to the TEM grid, initial thinning was performed at $30 \mathrm{kV}$ and $0.43 \mathrm{nA}$ on each side. The voltage was then lowered to $16 \mathrm{kV}$ to continue the thinning, and a final ion polishing was performed at $5 \mathrm{keV}$ and $0.68 \mathrm{nA}$ to remove damage surfaces caused by highervoltage milling. Subsequently, the FIB section was analyzed using the newly installed $200 \mathrm{keV} H i t a c h i$ HF5000 TEM/STEM. The HF5000 is equipped with a cold-field-emission gun with an energy resolution of $0.4 \mathrm{eV}$, a $3^{\text {rd }}$-order aberration-corrector for scanning TEM (STEM) imaging, a Gatan Quantum image filter (GIF) ER, and a 1.7-sr solid-angle Si-drift Oxford Twin EDS system. EELS spectra were acquired 
in diffraction mode (image coupled) and processed for background $\left(\mathrm{AE}^{-\mathrm{r}}\right)$ and the effects of multiple scattering (Fourier-ratio method).

We acquired X-ray elemental maps of a selected region in the FIB section (Fig. 1a-b). Apart from C in the capping layer, we did not detect any other C-rich material in the sample's region (Fig. 1b). Based on imaging and EDS mapping, we selected several areas, including the $\mathrm{C}$ capping layer, for more detailed analysis via EELS (Fig. 1c). We used DualEELS to simultaneously acquire the C K core-loss edge and low-loss region, including the zero-loss and plasmon peaks for the selected areas. The two EELS spectra in the $C$ capping layer reveal an energy-loss near-edge structure (ELNES) similar to amorphous C, i.e., they contain a sharp rise from edge onset to a peak at $285 \mathrm{eV}$ (the $\pi^{*}$ peak) followed by a delayed maximum beginning at $290 \mathrm{eV}$ (the $\sigma^{*}$ peak). This ELNES suggests the presence of aromatic functional groups in the capping layer. No other peaks were detected (Fig. 1c). In comparison, the ELNES for spectra acquired from the three other regions do not contain any detectable peaks within the energy region corresponding to the $\mathrm{C} \mathrm{K}$ absorption edge, even on the region directly adjacent to the $\mathrm{C}$ capping layer (e.g., area TB030_010; Fig. 1c). In summary, our preliminary data do not show any sign of C redeposition onto the FIB section, and thus suggest that $\mathrm{C}$ is suitable as a capping layer material during the preparation of FIB section containing C-bearing low-Z materials, such as organic matter in meteorites $[6]$.

\section{References:}

[1] G. Matrajt et al., Meteoritics and Planetary Science 43 (2008), p. 315-334.

[2] C. Vollmer et al., Proceedings of the National Academy of Sciences 111 (2014), p. 15338-15343.

[3] T. J. Zega et al., Meteoritics and Planetary Science 42 (2007), p. 1373-1386.

[4] C. Le Guillou et al., Geochimica et Cosmochimica Acta 131 (2014), p. 368-392.

[5] T. Yada et al., Meteoritics and Planetary Science 43 (2008), p. 1287-1298.

[6] This research is based upon work supported by NASA Grant NNX15AD94G for the NEXUS EOS program. TEM and FIB analyses were carried out at the University of Arizona Kuiper Core Imaging and Microscopy Facility (NSF Grant 1531243 and NASA Grants NNX15AJ22G and NNX12AL47G).
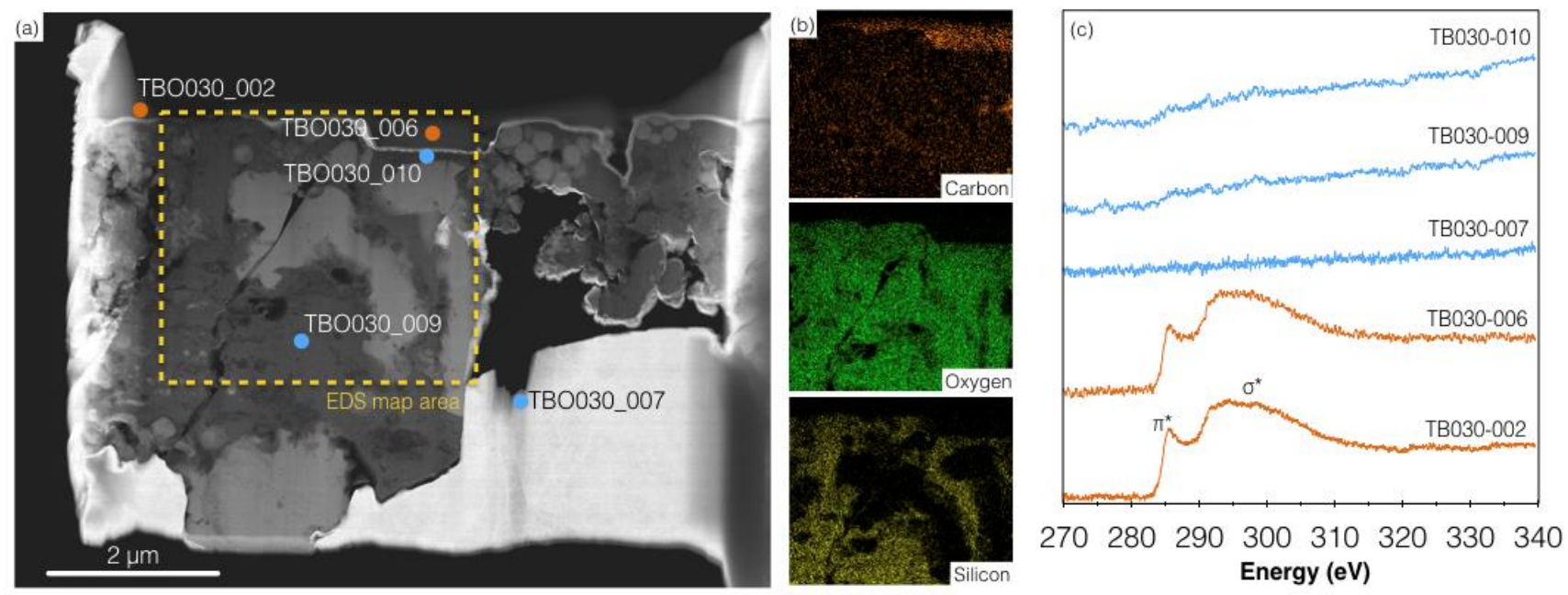

Figure 1. (a) Dark-field TEM image of the FIB section, with location of the EELS measurements (blue/orange dots). (b) EDS element maps (C, O, Si) of a selected area (see yellow rectangle for location in the section). (c) EELS spectra of the C capping layer (orange) and other areas (blue). 